\title{
A fractional step method for 2D parabolic convection-diffusion singularly perturbed problems: uniform convergence and order reduction
}

\author{
C. Clavero' ${ }^{1}$ (D) J. C. Jorge ${ }^{2}$
}

Received: 1 April 2016/ Accepted: 3 October 2016

(C) Springer Science+Business Media New York 2016

\begin{abstract}
In this work, we are concerned with the efficient resolution of two dimensional parabolic singularly perturbed problems of convection-diffusion type. The numerical method combines the fractional implicit Euler method to discretize in time on a uniform mesh and the classical upwind finite difference scheme, defined on a Shishkin mesh, to discretize in space. We consider general time-dependent Dirichlet boundary conditions, and we show that classical evaluations of the boundary conditions cause an order reduction in the consistency of the time integrator. An appropriate correction for the evaluations of the boundary data permits to remove such order reduction. Using this correction, we prove that the fully discrete scheme is uniformly convergent of first order in time and of almost first order in space. Some numerical experiments, which corroborate in practice the robustness and the efficiency of the proposed numerical algorithm, are shown; from them, we bring to light the influence in practice of the two options for the boundary data considered here, which is in agreement with the theoretical results.
\end{abstract}

Keywords Convection-diffusion · Singularly perturbed problems · Fractional euler method · Piecewise uniform meshes · Uniform convergence · Order reduction

Mathematics Subject Classification (2010) 65N05 - 65N06 $\cdot 65$ N10

C. Clavero

clavero@unizar.es

J. C. Jorge

jcjorge@upna.es

1 Department of Applied Mathematics and IUMA, University of Zaragoza, Zaragoza, Spain

2 Department of Computational and Mathematical Engineering, Public University of Navarra, Pamplona, Spain 


\section{Introduction}

Let us consider the initial and boundary value 2D time-dependent convectiondiffusion problem

$$
\begin{aligned}
& \mathcal{L} u \equiv \frac{\partial u}{\partial t}+\left(\mathcal{L}_{1, \varepsilon}(t)+\mathcal{L}_{2, \varepsilon}(t)\right) u=f, \text { in } \Omega \times(0, T], \\
& u(x, y, 0)=\varphi(x, y), \text { in } \Omega, \\
& u(x, y, t)=g(x, y, t), \text { in } \partial \Omega \times[0, T],
\end{aligned}
$$

where $\Omega \equiv(0,1) \times(0,1)$, and the spatial differential operators $\mathcal{L}_{i, \varepsilon}, i=1,2$ are given by

$$
\begin{aligned}
& \mathcal{L}_{1, \varepsilon}(t) \equiv-\varepsilon \frac{\partial^{2}}{\partial x^{2}}+v_{1}(x, y, t) \frac{\partial}{\partial x}+k_{1}(x, y, t), \\
& \mathcal{L}_{2, \varepsilon}(t) \equiv-\varepsilon \frac{\partial^{2}}{\partial y^{2}}+v_{2}(x, y, t) \frac{\partial}{\partial y}+k_{2}(x, y, t),
\end{aligned}
$$

respectively. We assume that the diffusion parameter $\varepsilon, 0<\varepsilon \leq 1$, can be very small, that the convective coefficients are strictly positive, i.e., $v_{i}(x, y, t) \geq \bar{v}>0$, and that the reaction terms satisfy $k_{i}(x, y, t) \geq 0, i=1,2$. Henceforth, we will suppose that enough smoothness and compatibility conditions among data hold in order to the solution is four times derivable in space and twice in time in the domain $\bar{\Omega} \times[0, T]$. In [6], the following sufficient conditions were given:

$$
\begin{aligned}
& f \in \mathcal{C}^{2+2 \alpha, 1+\alpha}(\bar{\Omega} \times[0, T]), \varphi \in \mathcal{C}^{4}(\bar{\Omega})(\alpha>0), \\
& \{g(0, y, t), g(1, y, t), g(x, 0, t), g(x, 1, t)\} \subset \mathcal{C}^{4,2}([0,1] \times[0, T]), \\
& g(x, y, 0)=\varphi(x, y), \quad(x, y) \in \partial \Omega, \\
& \frac{\partial g}{\partial t}(x, y, 0)=f(x, y, 0)-\left(\mathcal{L}_{1, \varepsilon}(0)+\mathcal{L}_{2, \varepsilon}(0)\right) \varphi(x, y), \quad(x, y) \in \partial \Omega, \\
& \frac{\partial^{2} g}{\partial t^{2}}(x, y, 0)=\frac{\partial f}{\partial t}(x, y, 0)-\left(\mathcal{L}_{1, \varepsilon}(0)+\mathcal{L}_{2, \varepsilon}(0)\right) f(x, y, 0) \\
& +\left(\mathcal{L}_{1, \varepsilon}(0)+\mathcal{L}_{2, \varepsilon}(0)\right)^{2} \varphi(x, y), \quad(x, y) \in \partial \Omega,
\end{aligned}
$$

and

$$
\begin{aligned}
\frac{\partial g}{\partial t}(x, y, t)+\left(\mathcal{L}_{1, \varepsilon}(t)+\mathcal{L}_{2, \varepsilon}\right)(t) g(x, y, t) & =f(x, y, t), \\
(x, y) & \in\{(0,0),(0,1),(1,0),(1,1)\}, t \in[0, T] .
\end{aligned}
$$

The numerical resolution of singularly perturbed problems is an interesting subject in applied mathematics which has been received many attention in the last years. To dispose of reliable numerical methods, fitted operator methods or fitted mesh methods are used (see $[9,11,14,16,17]$ and references therein). Specially interesting is the case when the problem is two dimensional in space of convection-diffusion 
type. The stationary case is considered, for instance, in $[4,10,12]$, and the timedependent problem is analyzed in [2, 3, 5]. In some of these works, it was described a two-step technique to construct the fully discrete scheme.

In [6], the authors consider and analyze the uniform convergence of a first stage of spatial semidiscretization, via an upwind finite difference method on a Shishkin mesh for a parabolic problem of convection-diffusion type; afterwards, the Euler implicit method completes the discretization. Using this method, the computational cost is large because pentadiagonal linear systems, one per time step, must be solved. This drawback is typical when classical implicit time integrators are used for solving, combined with finite differences, 2D parabolic problems. In [7], the reverse order was chosen, discretizing firstly in time and later on in space, for a parabolic problem of reaction-diffusion type; then, the use of an alternating direction method (see [18]), permits that only tridiagonal linear systems must be solved to obtain the numerical solution, which implies a remarkable cost reduction with respect to other, more classical, integration techniques. Here, we are going to extend the technique developed in [7] to the case of parabolic 2D convection-diffusion, but following the reverse order in the two-step technique considered in [5]. The reasons for choosing this option are that, firstly, the analysis of the uniform convergence of the fully discrete is simpler and, besides, that, in contrast with previous works (see for instance [3, 5]), no ratios between the discretization parameters are needed to prove the uniform convergence of the scheme.

Another interesting question, which is usually posed in the convergence analysis of numerical methods for parabolic problems, is related to the influence of the time-dependent boundary data of (1). It is well known (see, for instance $[1,15]$ and references therein) that, when using one step methods, a classical evaluation of the boundary conditions causes, in general, a reduction in the numerical order of convergence; this phenomenon is specially severe for most methods of alternating direction type. So, in this paper, we consider a different and simpler modification of these evaluations for the fractional implicit Euler method, which eliminates the loss of consistency without increasing the computational cost of the algorithm.

The rest of the paper is structured as follows. In Section 2, we introduce the spatial discretization of the continuous problem on a piecewise uniform mesh of Shishkin type, and we prove that it is uniformly convergent with respect to the diffusion parameter of almost first order. In Section 3, we introduce the time discretization proposed, by using the fractional implicit Euler method and proving that this time discretization is a first order uniformly convergent method. Also, we analyze the effect of two choices of evaluations of the data functions associated to the non-homogenous boundary conditions, in the uniform consistency of the method. Finally, in Section 4, some numerical results obtained for different test problems are shown; from them, we can observe the uniform convergent behavior of the numerical algorithm proposed here; as well, we show the effects of the classical evaluations of the boundary data and the improvement of them which we propose here.

Henceforth, $C$ denotes a generic positive constant independent of the diffusion parameter $\varepsilon$ and also of the discretization parameters $N$ and $M$. Below, we always use the pointwise maximum norm, denoted by $\|\cdot\|_{D}$ (where $D$ is the corresponding domain). 


\section{Spatial semidiscretization}

In this section, we define approximations of $u\left(x_{i}, y_{j}, t\right)$, where $\left(x_{i}, y_{j}\right)$ are going to be the grid points of a rectangular mesh $\bar{\Omega}_{N} \equiv I_{x, \varepsilon, N} \times I_{y, \varepsilon, N} \subset \bar{\Omega}$, which has $(N+1)^{2}$ nodes, for simplicity, we take the same number $N$ of subintervals in both space directions, and $t \in[0, T]$ remains as a continuous variable. We will denote $\Omega_{N}$ the subgrid of $\bar{\Omega}_{N}$ obtained by removing the points which belong to $\partial \Omega$.

Let us denote [.] $]_{N}$ the restriction of a function defined on $\bar{\Omega}$ to $\Omega_{N}, u_{N}(t)$, the semidiscrete grid functions which will approach $\left[u(x, y, t]_{N},[.]_{\bar{N}}\right.$, the restriction of a function defined on $\bar{\Omega}$ to $\bar{\Omega}_{N}$, and $u_{\bar{N}}(t)$ the semidiscrete grid functions which will approach $[u(x, y, t)]_{\bar{N}}$.

Typically, $u_{N}(t)$ is defined as the solution of a stiff Initial Value Problem which can be written in the form

$$
\begin{aligned}
& u_{N}^{\prime}(t)+\left(\mathcal{L}_{1, \varepsilon, N}(t)+\mathcal{L}_{2, \varepsilon, N}(t)\right) u_{\bar{N}}(t)=[f]_{N}, \\
& u_{\bar{N}}(t)=[g]_{\bar{N}} \text { in } \bar{\Omega}_{N} \backslash \Omega_{N}, \\
& u_{N}(0)=[\varphi]_{N},
\end{aligned}
$$

where $\mathcal{L}_{i, \varepsilon, N}, i=1,2$ must be appropriate spatial discretizations of the elliptic convection-diffusion operators $\mathcal{L}_{i, \varepsilon}, i=1,2$ given in (2).

Here, we consider the special mesh $\bar{\Omega}_{N} \equiv I_{x, \varepsilon, N} \times I_{y, \varepsilon, N}$, tensor product of one-dimensional meshes,

$$
I_{x, \varepsilon, N}=\left\{0=x_{0}<\ldots<x_{N}=1\right\}, I_{y, \varepsilon, N}=\left\{0=y_{0}<\ldots<y_{N}=1\right\},
$$

of Shishkin type as follows. We only give the details of the construction of $I_{x, \varepsilon, N}$ and analogously we proceed for $I_{y, \varepsilon, N}$.

Let us choose $N$ as an even number. We define the transition parameter

$$
\sigma_{x}=\min \left(1 / 2, m_{x} \varepsilon \ln N\right),
$$

where $m_{x} \geq 1 / \bar{v}$; then, the piecewise uniform mesh has $N / 2+1$ points in $\left[0,1-\sigma_{x}\right]$ and $\left[1-\sigma_{x}, 1\right]$. Therefore, the mesh points are given by

$$
x_{i}=\left\{\begin{array}{l}
2 i\left(1-\sigma_{x}\right) / N, i=0, \ldots, N / 2, \\
1-\sigma_{x}+2(i-N / 2) \sigma_{x} / N, i=N / 2+1, \ldots, N .
\end{array}\right.
$$

Using these meshes, $\mathcal{L}_{i, \varepsilon, N}, i=1,2$ are the discretization of the differential convection-diffusion operator $\mathcal{L}_{i, \varepsilon}, i=1,2$, via the simple upwind finite difference scheme, which is given by

$$
\begin{aligned}
& \mathcal{L}_{1, \varepsilon, N}(t) u_{N}(t)\left(x_{i}, y_{j}\right) \equiv l_{i-, j} u_{N}(t)\left(x_{i-1}, y_{j}\right)+l_{i+, j} u_{N}(t)\left(x_{i+1}, y_{j}\right) \\
& +l_{i, j}^{1} u_{N}(t)\left(x_{i}, y_{j}\right), \quad i=1, \ldots, N-1, j=0, \ldots, N,
\end{aligned}
$$

where

$$
\begin{aligned}
& l_{i-, j}=\frac{-\varepsilon}{h_{x, i} \tilde{h}_{x, i}}-\frac{v_{1}\left(x_{i}, y_{j}, t\right)}{h_{x, i}}, \quad l_{i+, j}=\frac{-\varepsilon}{h_{x, i+1} \tilde{h}_{x, i}}, \\
& l_{i, j}^{1}=-l_{i-, j}-l_{i+, j}+k_{1}\left(x_{i}, y_{j}, t\right),
\end{aligned}
$$


and analogously

$$
\begin{aligned}
& \mathcal{L}_{2, \varepsilon, N}(t) u_{N}(t)\left(x_{i}, y_{j}\right) \equiv l_{i, j-} u_{N}(t)\left(x_{i}, y_{j-1}\right)+l_{i, j+} u_{N}(t)\left(x_{i}, y_{j+1}\right) \\
& +l_{i, j}^{2} u_{N}(t)\left(x_{i}, y_{j}\right), \quad j=1, \ldots, N-1, i=0, \ldots, N,
\end{aligned}
$$

where

$$
\begin{aligned}
& l_{i, j-}=\frac{-\varepsilon}{h_{y, j} \tilde{h}_{y, j}}-\frac{v_{2}\left(x_{i}, y_{j}, t\right)}{h_{y, j}}, \quad l_{i, j+}=\frac{-\varepsilon}{h_{y, j+1} \tilde{h}_{y, j}}, \\
& l_{i, j}^{2}=-l_{i, j-}-l_{i, j+}+k_{2}\left(x_{i}, y_{j}, t\right),
\end{aligned}
$$

with $h_{x, i}=x_{i}-x_{i-1}, i=1, \ldots, N, h_{y, j}=y_{j}-y_{j-1}, j=1, \ldots, N, \tilde{h}_{x, i}=$ $\left(h_{x, i}+h_{x, i+1}\right) / 2, i=1, \ldots, N-1, \tilde{h}_{y, j}=\left(h_{y, j}+h_{y, j+1}\right) / 2, j=1, \ldots, N-1$.

Using this semidiscretization, in [6], the authors prove that the upwind scheme is an almost first-order uniformly convergent scheme; summarizing, under the smoothness assumptions made here, it holds that

$$
\left\|[u(x, y, t)]_{N}-u_{N}(t)\right\|_{\bar{\Omega}_{N}} \leq C N^{-1} \ln N, \quad \forall t \in(0, T] .
$$

\section{Time integration: unconditional convergence}

In this section, we introduce the numerical algorithm which we propose to integrate successfully the continuous problem (1). After having considered the spatial semidiscretization stage in the previous section, we complete the discretization process by using the fractional implicit Euler method as time integrator.

Using this method, we obtain numerical approximations $u_{N}^{m} \in R^{(N+1)^{2}}$ to $u_{N}\left(t_{m}\right)$, being $t_{m}=m \tau$, where $\tau=T / M$ is the chosen time step, which we consider constant for simplicity.

As a previous task, we need to establish the uniform behavior of the time derivatives and the fractional differentials of $u_{N}(t)$ involved in the analysis of the uniform consistency for the time integration process. In [6], the authors explain briefly that, under the smoothness and compatibility assumptions made here for the data of (1), it holds that $u_{N}(t) \in\left(C^{2}[0, T]\right)^{(N+1)^{2}}$, and its second derivative is bounded independently of $\varepsilon$. From this property, joint to the smoothness assumptions made on $f$, it is immediate to deduce that the elementary differentials $L_{\varepsilon, N} u_{N}(t), L_{\varepsilon, N}^{2} u_{N}(t)$ are bounded independently of $\varepsilon$. These differentials are typically involved in the consistency analysis of classical one step time integrators, for instance, Runge-Kutta methods. In this paper, we will assume a similar but extra natural condition in this context which is that the fractionated elementary differentials $L_{i, \varepsilon, N} u_{N}(t), L_{i, \varepsilon, N} L_{j, \varepsilon, N} u_{N}(t), i, j=1,2$, satisfy

$$
\left\|L_{i, \varepsilon, N} u_{N}(t)\right\|_{\Omega_{N}} \leq C,\left\|L_{i, \varepsilon, N} L_{j, \varepsilon, N} u_{N}(t)\right\|_{\Omega_{N}} \leq C, i, j=1,2 .
$$

This property is easily deduced in the cases that operators $L_{i, \varepsilon, N}, i=1,2$ commute. In such cases, these fractional elementary differentials can be described as solutions of Initial Value Problems similar to (15), where all of the data functions 
are $\varepsilon$-uniformly bounded. For example, $\omega \equiv L_{1, \varepsilon, N} u_{N}(t)$ can be described as the solution of

$$
\begin{aligned}
& \omega^{\prime}(t)+\left(\mathcal{L}_{1, \varepsilon, N}(t)+\mathcal{L}_{2, \varepsilon, N}(t)\right) \omega(t)=L_{1, \varepsilon, N}[f]_{N}, \\
& \omega(t)=\tilde{g}_{\bar{N}} \text { in } \bar{\Omega}_{N} \backslash \Omega_{N}, \\
& \omega(0)=L_{1, \varepsilon, N}[\varphi]_{N},
\end{aligned}
$$

being $\tilde{g}_{\bar{N}}=L_{1, \varepsilon, N}[g]_{\bar{N}}$ for $y \in\{0,1\}$ and $\tilde{g}_{\bar{N}}=[f]_{\bar{N}}-\left[\frac{\partial g}{\partial t}\right]_{\bar{N}}-L_{2, \varepsilon, N}[g]_{\bar{N}}$ for $x \in\{0,1\}$.

\subsection{The fully discrete scheme}

In this section, we describe the numerical method which we propose to discretize in time the Initial Value Problems (15). We consider the fractional implicit Euler method (see [5, 18]), which can be written as a two half step scheme as follows. Let $\tau \equiv T / M$ be the time step, and let us consider the mesh $\bar{I}_{M}=\left\{t_{m}=m \tau, m=\right.$ $0,1, \ldots, M\}$. Let $u_{N}^{m} \approx u_{N}\left(x, y, t_{m}\right), m=0,1, \ldots, M$, be the numerical solutions defined as follows:

i) (initialize)

$u_{N}^{0}=[\varphi(x, y)]_{N}$, in $\Omega_{N}$.

$u_{\bar{N}}^{0}=[g(x, y, 0)]_{\bar{N}}$, in $\Omega_{\bar{N}} \backslash \Omega_{N}$.

ii) (first half step)

$\left(I+\tau \mathcal{L}_{1, \varepsilon, N}\left(t_{m+1}\right)\right) u_{\bar{N}}^{m+1 / 2}=u_{\frac{m}{N}}^{+} \tau f_{1, \bar{N}}^{m+1}$, in $\Omega_{\bar{N}} \backslash\{0,1\} \times[0,1]$,

$u_{\bar{N}}^{m+1 / 2}=g_{\bar{N}}^{m+1 / 2}$, in $\Omega_{\bar{N}} \cap\{0,1\} \times[0,1]$.

iii) (second half step)

$$
\begin{aligned}
& \left(I+\tau \mathcal{L}_{2, \varepsilon, N}\left(t_{m+1}\right)\right) u_{\bar{N}}^{m+1}=u_{\bar{N}}^{m+1 / 2}+\tau f_{2, \bar{N}}^{m+1}, \text { in } \Omega_{\bar{N}} \backslash[0,1] \times\{0,1\},, \\
& u_{\bar{N}}^{m+1}(x, y)=g_{\bar{N}}^{m+1}, \text { in } \Omega_{\bar{N}} \cap[0,1] \times\{0,1\}, \\
& m=0, \ldots, M-1,
\end{aligned}
$$

being

$$
f=f_{1}+f_{2}, f_{1, \bar{N}}^{m+1}=\left[f_{1}\left(x, y, t_{m+1}\right)\right]_{\bar{N}}, f_{2, \bar{N}}^{m+1}=\left[f_{2}\left(x, y, t_{m+1}\right)\right]_{\bar{N}} .
$$

Of course, as a typical advantage of Alternating Direction methods, the resolution of the half steps involves a set of $N+1$ uncoupled linear tridiagonal systems of dimension $N-1$ which can be solved in parallel. Moreover, the advance of one step in time can be done with a number of arithmetic operations of size $\mathcal{O}\left(N^{2}\right)$ which is optimal in terms of the computational complexity of the method.

In the literature (see $[1,13,15])$, the most classical option for the evaluations of the boundary data is given by

$$
\begin{aligned}
& g_{\bar{N}}^{m+1 / 2}=\left[g\left(x, y, t_{m+1}\right)\right]_{\bar{N}}, \text { in } \Omega_{\bar{N}} \cap\{0,1\} \times[0,1], \\
& g_{\bar{N}}^{m+1}=\left[g\left(x, y, t_{m+1}\right)\right]_{\bar{N}}, \text { in } \Omega_{\bar{N}} \cap[0,1] \times\{0,1\} .
\end{aligned}
$$


This option is natural in the sense that, classically, the contributions of the boundary data are added to the source term $[f(x, y, t)]_{N}$ before the time integration process. Nevertheless, in most of cases, this choice reduces the order of unconditional (independent of $N$ ) consistency to zero and causes a sharp increase in the global error of the method as well as strong difficulties for proving its uniform convergence.

Here, we propose a different choice for the boundary data, given by

$$
\begin{aligned}
& g_{\bar{N}}^{m+1 / 2}=\left(I+\tau \mathcal{L}_{2, \varepsilon, N}\left(t_{m+1}\right)\left[g\left(x, y, t_{m+1}\right)\right]_{\bar{N}}-\tau f_{2, \bar{N}}^{m+1}, \text { in } \Omega_{\bar{N}} \cap\{0,1\} \times[0,1],\right. \\
& g_{\bar{N}}^{m+1}=\left[g\left(x, y, t_{m+1}\right)\right]_{\bar{N}}, \text { in } \Omega_{\bar{N}} \cap[0,1] \times\{0,1\} .
\end{aligned}
$$

\subsection{Stability}

Let us define the operators $\left(I+\tau \mathcal{L}_{1, \varepsilon, N, 0}(t)\right)^{-1}$ (analogously $\left.\left(I+\tau \mathcal{L}_{2, \varepsilon, N, 0}(t)\right)^{-1}\right)$, as follows. Let $v_{N}=\left(I+\tau \mathcal{L}_{1, \varepsilon, N, 0}^{n}(t)\right)^{-1} u_{N}$ be the solution of the linear system

$$
\begin{aligned}
& \left(I+\tau \mathcal{L}_{1, \varepsilon, N}^{n}(t)\right) v_{N}=u_{N}, \quad(x, y) \text { in } \Omega_{N}, \\
& v_{\bar{N}}=0, \text { in } \Omega_{\bar{N}} \backslash \Omega_{N} .
\end{aligned}
$$

From classical properties of $M$-matrices (see, e.g., [14]), it is straightforward to deduce

$$
\left\|\left(I+\tau \mathcal{L}_{i, \varepsilon, N, 0}(t)\right)^{-1}\right\|_{\infty} \leq 1, \quad i=1,2 .
$$

\subsection{Uniform and unconditional consistency}

Let us define $e_{N}^{m+1}$ the local error in time of the scheme (16) at $t_{m+1}$ as follows:

$$
e_{N}^{m+1}=u_{N}\left(t_{m+1}\right)-\tilde{u}_{N}^{m+1}
$$

where $\tilde{u}_{N}^{m+1}$ is the solution of the auxiliary problem

$$
\begin{aligned}
& \left(I+\tau \mathcal{L}_{1, \varepsilon, N}\left(t_{m+1}\right)\right) \tilde{u}_{N}^{m+1 / 2}=\left[u\left(t_{m}\right)\right]_{N}^{m}+\tau f_{1, N}^{m+1}, \text { in } \Omega_{\bar{N}} \backslash\{0,1\} \times[0,1], \\
& \tilde{u}_{\bar{N}}^{m+1 / 2}=g_{\bar{N}}^{m+1 / 2}, \text { in } \Omega_{\bar{N}} \cap\{0,1\} \times[0,1], \\
& \left(I+\tau \mathcal{L}_{2, \varepsilon, N}\left(t_{m+1}\right)\right) \tilde{u}_{N}^{m+1}=\tilde{u}_{N}^{m+1 / 2}+\tau f_{2, N}^{m+1}, \text { in } \Omega_{\bar{N}} \backslash[0,1] \times\{0,1\}, \\
& \tilde{u}_{N}^{m+1}(x, y)=g_{\bar{N}}^{m+1}, \text { in } \Omega_{\bar{N}} \cap[0,1] \times\{0,1\}, \\
&
\end{aligned}
$$

Lemma 1 Under the assumptions (3), (4), (5) and (14), if we choose the boundary data given in (19), then the local error defined by (22), (23) satisfies

$$
\left\|e_{N}^{m}\right\|_{\Omega_{N}} \leq C \tau^{2}, \quad m=1, \ldots, M
$$

and therefore (16) is a first order uniformly consistent method. 
Proof From the definition of $\tilde{u}_{N}^{m+1}$ given in (23), it is easily deduced that in $\Omega_{N}$ it holds

$$
\left(I+\tau \mathcal{L}_{1, \varepsilon, N}\left(t_{m+1}\right)\left(\left(I+\tau \mathcal{L}_{2, \varepsilon, N}\left(t_{m+1}\right)\right) \tilde{u}_{N}^{m+1}-\tau f_{2, N}^{m+1}\right)\right)=\left[u\left(t_{m}\right)\right]_{N}+\tau f_{1, N}^{m+1} .
$$

On the other hand, by using the simple Taylor expansion

$$
u_{N}\left(t_{m}\right)=u_{N}\left(t_{m+1}\right)-\tau u_{N}^{\prime}\left(t_{m+1}\right)+\mathcal{O}\left(\tau^{2}\right),
$$

and taking into account that $u_{N}^{\prime}\left(t_{m+1}\right)=f_{1, N}^{m+1}+f_{2, N}^{m+1}-\mathcal{L}_{1, \varepsilon, N}\left(t_{m+1}\right) u_{N}\left(t_{m+1}\right)-$ $\mathcal{L}_{2, \varepsilon, n}\left(t_{m+1}\right) u_{N}\left(t_{m+1}\right)$, it can be deduced that

$$
\begin{aligned}
& \left(I+\tau \mathcal{L}_{1, \varepsilon, N}\left(t_{m+1}\right)\right)\left(\left(I+\tau \mathcal{L}_{2, \varepsilon, N}\left(t_{m+1}\right)\right) u_{N}\left(t_{m+1}\right)-\tau f_{2, N^{m+1}}\right) \\
& =u_{N}\left(t_{m}\right)+\tau f_{1, N}^{m+1}+\mathcal{O}\left(\tau^{2}\right) .
\end{aligned}
$$

Subtracting (26) and (25), it holds

$$
\left(I+\tau \mathcal{L}_{1, \varepsilon, N}\left(t_{m+1}\right)\right)\left(I+\tau \mathcal{L}_{2, \varepsilon, N}\left(t_{m+1}\right)\right) e_{N}^{m+1}=\mathcal{O}\left(\tau^{2}\right) .
$$

Regarding to the boundary data, it is trivial that $u_{\bar{N}}\left(t_{m+1}\right)=g_{\bar{N}}^{m+1}$ in $\Omega_{\bar{N}} \cap[0,1] \times$ $\{0,1\}$, and it is also clear that, as we have chosen

$$
g_{\bar{N}}^{m+1 / 2}=\left(I+\tau \mathcal{L}_{2, \varepsilon, N}\left(t_{m+1}\right)\right) u_{\bar{N}}\left(t_{m+1}\right)-\tau f_{2, \bar{N}}^{m+1} \text { in } \Omega_{\bar{N}} \cap\{0,1\} \times[0,1],
$$

the local error can be written as the solution of a problem of the form

$$
\begin{aligned}
& \left(I+\tau \mathcal{L}_{1, \varepsilon, N}\left(t_{m+1}\right)\right) e_{\bar{N}}^{m+1 / 2}=\mathcal{O}\left(\tau^{2}\right), \quad \text { in } \Omega_{N}, \\
& e_{\bar{N}}^{m+1 / 2}=0, \text { in } \Omega_{\bar{N}} \backslash \Omega_{N}, \\
& \left(I+\tau \mathcal{L}_{2, \varepsilon, N}^{n+1}\left(t_{m+1}\right)\right) e_{\bar{N}}^{m+1}=e_{\bar{N}}^{m+1 / 2}, \text { in } \Omega_{N}, \\
& e_{\bar{N}}^{m+1}(x, y)=0, \text { in } \Omega_{\bar{N}} \backslash \Omega_{N} .
\end{aligned}
$$

From (28) and the stability property (21), the required result (24) follows.

Remark 1 Note that for non-homogeneous boundary data $g(x, y, t)$, in general $\mathcal{L}_{2, \varepsilon, N}\left(t_{m+1}\right)\left[g\left(x, y, t_{m+1}\right)\right]_{\bar{N}}-f_{2, \bar{N}}^{m+1} \neq 0$. Therefore, a term of size $O(\tau)$ appears in the difference between the natural boundary data given by $\left[g\left(x, y, t_{m+1}\right)\right]_{\bar{N}}$, and those ones considered here $\left(g_{\bar{N}}^{m+1 / 2}\right)$ for the first half step. This fact causes an order reduction in the order of consistency, up to order 0 if the classical evaluations of the boundary data are chosen.

Remark 2 In [3], the authors propose and analyze a similar algorithm for a less general problem; therein, the boundary conditions are homogeneous. In that paper, to avoid the order reduction, a suitable splitting $f=f_{1}+f_{2}$, in such way that $f_{2}=0$ in $\{0,1\} \times[0,1] \times[0, T]$, was necessary to complete the analysis. This restriction agrees completely with the analysis for the (more general) case which we have studied here. In fact, with the boundary data proposed in (19) any smooth splitting for $f$ can be considered without risk of order reduction. 


\subsection{Uniform convergence of the time integration}

Let us introduce the global error of the scheme (16) at time $t_{m}$ as usual, i.e., $E_{N}^{m} \equiv$ $u_{N}\left(t_{m}\right)-u_{N}^{m}$.

Theorem 1 Under the assumptions (3), (4), (5) and (14), if we choose the boundary data given in (19), then it holds

$$
\left\|E_{N}^{m}\right\|_{\Omega_{N}} \leq C \tau, \forall m=1, \ldots, M,
$$

i. e., the time integration process (16) is uniformly and unconditionally convergent of first order.

Proof Subtracting and adding $\tilde{u}_{N}^{m}$, trivially we have

$$
E_{N}^{m}=e_{N}^{m}+\tilde{u}_{N}^{m}-u_{N}^{m}=e_{N}^{m}+\left(I+\tau \mathcal{L}_{2, \varepsilon, N, 0}\left(t_{m}\right)\right)^{-1}\left(I+\tau \mathcal{L}_{1, \varepsilon, N, 0}^{n}\left(t_{m}\right)\right)^{-1} E^{n-1} .
$$

Using this recurrence, combined with (21) and (24), the result trivially follows.

\subsection{Uniform convergence of the algorithm}

Theorem 2 Assuming (3), (4), (5), and (14), if we use the improved boundary data (19), then the global error given by

$$
E_{N, M} \equiv \max _{1 \leq m \leq M}\left\|\left[u\left(x, y, t_{m}\right)\right]_{N}-u_{N}^{m}\right\|_{\Omega_{N}},
$$

satisfies

$$
E_{N, M} \leq C\left(N^{-1} \ln N+M^{-1}\right) .
$$

Thus, the proposed method is unconditionally and uniformly convergent of first order in time and almost first order in space, up to a typical logarithmic factor.

Proof We only need to add and subtract the term $u_{N}\left(t_{m}\right)$ and use the triangle inequality to deduce

$$
E_{N, M} \leq \max _{1 \leq m \leq M}\left\|\left[u\left(x, y, t_{m}\right)\right]_{N}-u_{N}\left(t_{m}\right)\right\|_{\Omega_{N}}+\max _{1 \leq m \leq M}\left\|E_{N}^{m}\right\|_{\Omega_{N}} .
$$

Finally, from (13) and (29), the result follows.

\section{Numerical experiments}

In this section, we solve some test problems using our numerical algorithm. The first example is given by

$$
\begin{aligned}
& u_{t}-\varepsilon \Delta u+(2 x+1) u_{x}+(2 y+1) u_{y}+30 u=f(x, y, t),(x, y, t) \in \Omega \times[0,1], \\
& u(x, y, t)=g(x, y, t), \text { in } \partial \Omega \times[0,1] \\
& u(x, y, 0)=\varphi(x, y), \quad x, y \in[0,1],
\end{aligned}
$$


where $f(x, y, t), g(x, y, t)$ and $\varphi(x, y)$ are chosen in such way that the exact solution is $u(x, y, t)=\left(1-e^{-30 t}\right)(\Psi(x) \Psi(y)-x y)$, with

$$
\Psi(z) \equiv z+\frac{e^{-\frac{2}{\varepsilon}}-e^{\frac{z^{2}+z-2}{\varepsilon}}}{1-e^{-\frac{2}{\varepsilon}}} .
$$

Figure 1 shows the solution at the final time $t=1$; from it, we clearly see the boundary layers at $x=1$ and $y=1$.

In all tables corresponding to example (31), we take $m_{x}=m_{y}=1$ to define the transition parameters of the meshes $I_{1, \varepsilon, N}$ and $I_{2, \varepsilon, N}$, respectively. Moreover, we decompose (see [3]) the right-hand side in the form $f(x, y, t)=$ $f_{1}(x, y, t)+f_{2}(x, y, t)$, where $f_{2}(x, y, t)=f(x, 0, t)+y(f(x, 1, t)-f(x, 0, t))$ and $f_{1}(x, y, t)=f(x, y, t)-f_{2}(x, y, t)$.

As the exact solution is known, the maximum global errors at the mesh points can be computed exactly by

$$
e_{N, M}=\max _{0 \leq n \leq M} \max _{0 \leq i \leq N} \max _{0 \leq j \leq N}\left|U_{N}^{n}-u\left(x_{i}, y_{j}, t_{n}\right)\right|,
$$

and therefore the numerical orders of convergence are calculated by

$$
p=\log \left(e_{N, M} / e_{2 N, 2 M}\right) / \log 2 .
$$

From these values, we calculate the uniform maximum errors by

$$
\operatorname{emax}^{N, M}=\max _{\varepsilon} e_{N, M}
$$

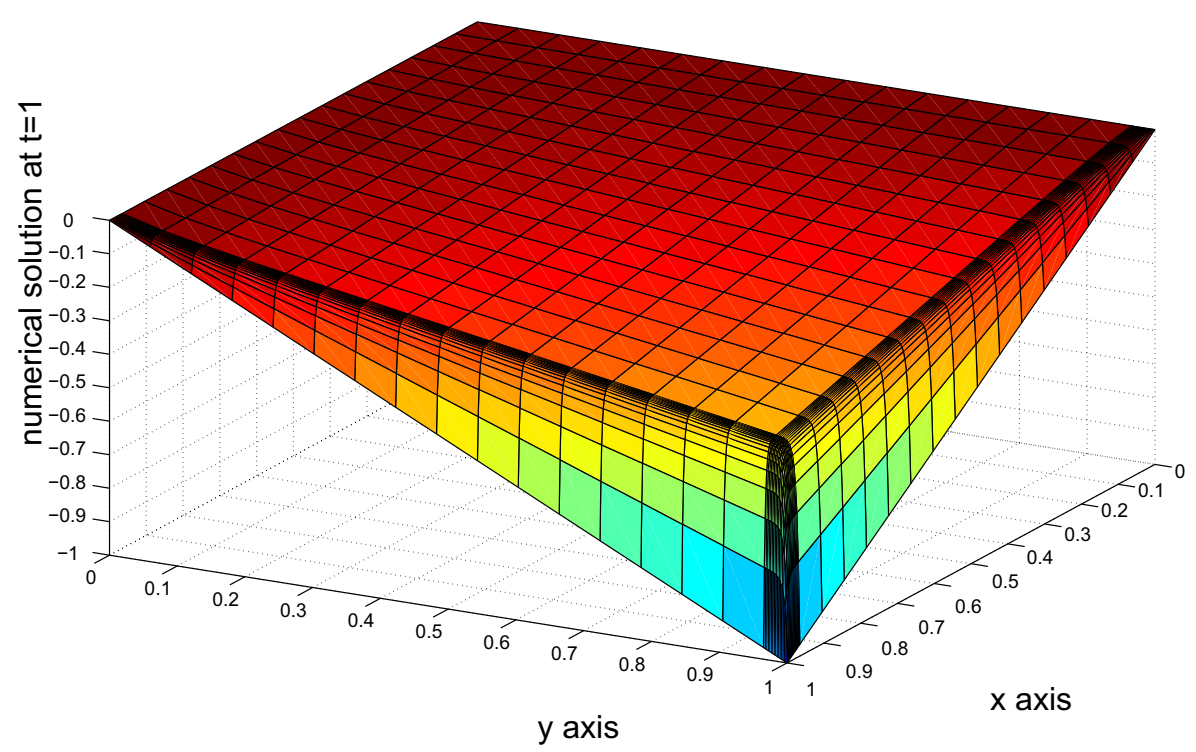

Fig. 1 Numerical solution of example (31) for $\varepsilon=10^{-2}, N=M=32$, at the final time $t=1$ 
and from them, in a usual way, the corresponding numerical uniform orders of convergence are given by

$$
p^{\text {uni }}=\log \left(\operatorname{emax}^{N, M} / \operatorname{emax}^{2 N, 2 M}\right) / \log 2 .
$$

Table 1 displays the results for problem (31) by using our fully discrete scheme when the classical boundary conditions are used. From them, we observe a reduction in the numerical orders of convergence.

Next, we consider the choice the boundary data given in (19). Table 2 displays the results for problem (31) by using this option. From it, an almost first order uniformly convergent numerical behavior is observed. Moreover, note that the maximum errors for any value of $\varepsilon$ are smaller than those ones given in Table 1. A similar behavior has been observed in all of the experiments which we have performed.

Nevertheless, in the case of using natural boundary data, the global errors behave better than expected; this phenomenon has been deeply studied in the numerical integration of canonical parabolic problems like the heat equation, where several authors have proven that one order of convergence can be recovered many times, by using a summation by parts reasoning. This technique, which has been successfully used for studying the integration of simple parabolic problems (see, e.g., $[1,13,15]$ ), has not been used in the context of parabolic singularly perturbed problems and

Table 1 Maximum errors and orders of convergence for (31) with natural boundary conditions

\begin{tabular}{|c|c|c|c|c|c|}
\hline$\varepsilon$ & $\begin{array}{l}\mathrm{N}=16 \\
\mathrm{M}=8\end{array}$ & $\begin{array}{l}\mathrm{N}=32 \\
\mathrm{M}=16\end{array}$ & $\begin{array}{l}\mathrm{N}=64 \\
\mathrm{M}=32\end{array}$ & $\begin{array}{l}N=128 \\
M=64\end{array}$ & $\begin{array}{l}\mathrm{N}=256 \\
\mathrm{M}=128\end{array}$ \\
\hline $2^{-6}$ & $\begin{array}{l}8.9908 \mathrm{E}-1 \\
0.503\end{array}$ & $\begin{array}{l}6.3453 \mathrm{E}-1 \\
0.641\end{array}$ & $\begin{array}{l}4.0693 \mathrm{E}-1 \\
0.651\end{array}$ & $\begin{array}{l}2.5911 \mathrm{E}-1 \\
0.681\end{array}$ & $1.6161 \mathrm{E}-1$ \\
\hline $2^{-8}$ & $\begin{array}{l}9.3261 \mathrm{E}-1 \\
0.505\end{array}$ & $\begin{array}{l}6.5698 \mathrm{E}-1 \\
0.643\end{array}$ & $\begin{array}{l}4.2064 \mathrm{E}-1 \\
0.640\end{array}$ & $\begin{array}{l}2.6994 \mathrm{E}-1 \\
0.685\end{array}$ & $1.6796 \mathrm{E}-1$ \\
\hline $2^{-10}$ & $\begin{array}{l}9.4281 \mathrm{E}-1 \\
0.507\end{array}$ & $\begin{array}{l}6.6341 \mathrm{E}-1 \\
0.644\end{array}$ & $\begin{array}{l}4.2459 \mathrm{E}-1 \\
0.635\end{array}$ & $\begin{array}{l}2.7338 \mathrm{E}-1 \\
0.685\end{array}$ & $1.7008 \mathrm{E}-1$ \\
\hline $2^{-12}$ & $\begin{array}{l}9.4556 \mathrm{E}-1 \\
0.507\end{array}$ & $\begin{array}{l}6.6516 \mathrm{E}-1 \\
0.644\end{array}$ & $\begin{array}{l}4.2567 \mathrm{E}-1 \\
0.634\end{array}$ & $\begin{array}{l}2.7438 \mathrm{E}-1 \\
0.685\end{array}$ & $1.7070 \mathrm{E}-1$ \\
\hline $2^{-14}$ & $\begin{array}{l}9.4625 \mathrm{E}-1 \\
0.508\end{array}$ & $\begin{array}{l}6.6561 \mathrm{E}-1 \\
0.644\end{array}$ & $\begin{array}{l}4.2595 \mathrm{E}-1 \\
0.633\end{array}$ & $\begin{array}{l}2.7465 \mathrm{E}-1 \\
0.685\end{array}$ & $1.7088 \mathrm{E}-1$ \\
\hline $2^{-16}$ & $\begin{array}{l}9.4642 \mathrm{E}-1 \\
0.508\end{array}$ & $\begin{array}{l}6.6573 \mathrm{E}-1 \\
0.644\end{array}$ & $\begin{array}{l}4.2602 \mathrm{E}-1 \\
0.633\end{array}$ & $\begin{array}{l}2.7473 \mathrm{E}-1 \\
0.685\end{array}$ & $1.7092 \mathrm{E}-1$ \\
\hline- & $\begin{array}{l}- \\
-\end{array}$ & $\begin{array}{l}- \\
-\end{array}$ & $\begin{array}{l}- \\
-\end{array}$ & $\begin{array}{l}- \\
-\end{array}$ & - \\
\hline $2^{-26}$ & $\begin{array}{l}9.4647 \mathrm{E}-1 \\
0.508\end{array}$ & $\begin{array}{l}6.6577 \mathrm{E}-1 \\
0.644\end{array}$ & $\begin{array}{l}4.2604 \mathrm{E}-1 \\
0.633\end{array}$ & $\begin{array}{l}2.7476 \mathrm{E}-1 \\
0.685\end{array}$ & $1.7094 \mathrm{E}-1$ \\
\hline $\operatorname{emax}^{N, M}$ & $9.4647 \mathrm{E}-1$ & $6.6577 \mathrm{E}-1$ & $4.2604 \mathrm{E}-1$ & $2.7476 \mathrm{E}-1$ & $1.7094 \mathrm{E}-1$ \\
\hline$p^{\text {uni }}$ & 0.508 & 0.644 & 0.633 & 0.685 & \\
\hline
\end{tabular}


Table 2 Maximum errors and orders of convergence for (31) with improved boundary conditions

\begin{tabular}{|c|c|c|c|c|c|}
\hline$\varepsilon$ & $\mathrm{N}=16$ & $\mathrm{~N}=32$ & $\mathrm{~N}=64$ & $\mathrm{~N}=128$ & $N=256$ \\
\hline & $M=8$ & $M=16$ & $\mathrm{M}=32$ & $M=64$ & $M=128$ \\
\hline \multirow[t]{2}{*}{$2^{-6}$} & $8.4881 \mathrm{E}-1$ & $5.8678 \mathrm{E}-1$ & $3.6410 \mathrm{E}-1$ & $2.0838 \mathrm{E}-1$ & $1.1328 \mathrm{E}-1$ \\
\hline & 0.533 & 0.688 & 0.805 & 0.879 & \\
\hline \multirow[t]{2}{*}{$2^{-8}$} & $8.8872 \mathrm{E}-1$ & $6.0980 \mathrm{E}-1$ & $3.7657 \mathrm{E}-1$ & $2.1503 \mathrm{E}-1$ & $1.1683 \mathrm{E}-1$ \\
\hline & 0.543 & 0.695 & 0.808 & 0.880 & \\
\hline \multirow[t]{2}{*}{$2^{-10}$} & $9.0122 \mathrm{E}-1$ & $6.1653 \mathrm{E}-1$ & $3.7993 \mathrm{E}-1$ & $2.1676 \mathrm{E}-1$ & $1.1776 \mathrm{E}-1$ \\
\hline & 0.548 & 0.698 & 0.810 & 0.880 & \\
\hline \multirow[t]{2}{*}{$2^{-12}$} & $9.0470 \mathrm{E}-1$ & $6.1830 \mathrm{E}-1$ & $3.8080 \mathrm{E}-1$ & $2.1720 \mathrm{E}-1$ & $1.1799 \mathrm{E}-1$ \\
\hline & 0.549 & 0.699 & 0.810 & 0.880 & \\
\hline \multirow[t]{2}{*}{$2^{-14}$} & $9.0562 \mathrm{E}-1$ & $6.1874 \mathrm{E}-1$ & $3.8102 \mathrm{E}-1$ & $2.1731 \mathrm{E}-1$ & $1.1805 \mathrm{E}-1$ \\
\hline & 0.550 & 0.699 & 0.810 & 0.880 & \\
\hline \multirow[t]{2}{*}{$2^{-16}$} & $9.0585 \mathrm{E}-1$ & $6.1885 \mathrm{E}-1$ & $3.8107 \mathrm{E}-1$ & $2.1734 \mathrm{E}-1$ & $1.1806 \mathrm{E}-1$ \\
\hline & 0.550 & 0.700 & 0.810 & 0.880 & \\
\hline \multirow[t]{2}{*}{-} & - & - & - & - & - \\
\hline & - & - & - & - & \\
\hline \multirow[t]{2}{*}{$2^{-26}$} & $9.0592 \mathrm{E}-1$ & $6.1889 \mathrm{E}-1$ & $3.8109 \mathrm{E}-1$ & $2.1735 \mathrm{E}-1$ & $1.1807 \mathrm{E}-1$ \\
\hline & 0.550 & 0.700 & 0.810 & 0.880 & \\
\hline $\operatorname{emax}^{N, M}$ & $9.0592 \mathrm{E}-1$ & $6.1889 \mathrm{E}-1$ & $3.8109 \mathrm{E}-1$ & $2.1735 \mathrm{E}-1$ & $1.1807 \mathrm{E}-1$ \\
\hline$p^{\text {uni }}$ & 0.550 & 0.700 & 0.810 & 0.880 & \\
\hline
\end{tabular}

seems complicated to apply it. Thus, we can conclude that the new proposal provides improvements both from theoretical and practical points of view.

In order to clarify a bit more the influence, in the numerical behavior of the method, of the two options for the boundary data considered here as well as the improvements provided by the non natural evaluations of the boundary conditions, we estimate the local errors in time. As the exact solution is known, these estimates can be approximated by

$$
\tilde{e}_{N, M}=\max _{0 \leq m \leq M} \max _{0 \leq i \leq N} \max _{0 \leq j \leq N}\left|\tilde{U}_{N}^{m}-u\left(x_{i}, y_{j}, t_{m}\right)\right| .
$$

where $N$ must be chosen large enough in order to the contribution of the spatial discretization can be neglected. From them, we obtain the quantities

$$
\tilde{p}=\log \left(\tilde{e}_{N, M} / \tilde{e}_{N, 2 M}\right) / \log 2,
$$

and note that the corresponding numerical orders of consistency are given by $\tilde{p}-1$.

In next tables, we show such estimated local errors and the values of $\tilde{p}$ corresponding to the two choices of the boundary data, taking $N=512$ fixed. Table 3 displays the estimated local errors when natural boundary conditions are chosen. Here, uniform consistency of order zero is observed.

Table 4 displays the local errors obtained when the improved boundary conditions are considered. Note that the local errors are substantially smaller than in the previous 
Table 3 Local errors and values of $\tilde{p}$ for (31) with natural boundary conditions, $N=512$

\begin{tabular}{llllll}
\hline$\varepsilon$ & $\mathrm{M}=8$ & $\mathrm{M}=16$ & $\mathrm{M}=32$ & $\mathrm{M}=64$ & $\mathrm{M}=128$ \\
\hline $2^{-6}$ & $7.7818 \mathrm{E}-1$ & $5.5586 \mathrm{E}-1$ & $4.2260 \mathrm{E}-1$ & $2.8458 \mathrm{E}-1$ & $1.7001 \mathrm{E}-1$ \\
& 0.485 & 0.395 & 0.570 & 0.743 & \\
$2^{-8}$ & $8.0824 \mathrm{E}-1$ & $5.7012 \mathrm{E}-1$ & $4.3375 \mathrm{E}-1$ & $2.9248 \mathrm{E}-1$ & $1.7518 \mathrm{E}-1$ \\
& 0.504 & 0.394 & 0.569 & 0.739 & \\
$2^{-10}$ & $8.1763 \mathrm{E}-1$ & $5.7464 \mathrm{E}-1$ & $4.3731 \mathrm{E}-1$ & $2.9506 \mathrm{E}-1$ & $1.7694 \mathrm{E}-1$ \\
& 0.509 & 0.394 & 0.568 & 0.738 & \\
$2^{-12}$ & $8.2041 \mathrm{E}-1$ & $5.7599 \mathrm{E}-1$ & $4.3837 \mathrm{E}-1$ & $2.9583 \mathrm{E}-1$ & $1.7746 \mathrm{E}-1$ \\
& 0.510 & 0.394 & 0.567 & 0.737 & \\
$2^{-14}$ & $8.2120 \mathrm{E}-1$ & $5.7637 \mathrm{E}-1$ & $4.3868 \mathrm{E}-1$ & $2.9605 \mathrm{E}-1$ & $1.7761 \mathrm{E}-1$ \\
& 0.511 & 0.394 & 0.567 & 0.737 & \\
$2^{-16}$ & $8.2142 \mathrm{E}-1$ & $5.7649 \mathrm{E}-1$ & $4.3876 \mathrm{E}-1$ & $2.9611 \mathrm{E}-1$ & $1.7765 \mathrm{E}-1$ \\
& 0.511 & 0.394 & 0.567 & 0.737 & \\
- & - & - & - & - & - \\
& - & - & - & - & $1.7767 \mathrm{E}-1$ \\
$2^{-26}$ & $8.2150 \mathrm{E}-1$ & $5.7653 \mathrm{E}-1$ & $4.3880 \mathrm{E}-1$ & $2.9613 \mathrm{E}-1$ & \\
& 0.511 & 0.394 & 0.567 & 0.737 & \\
& & & & &
\end{tabular}

table, where the classical boundary data are used and also that the orders of consistency are higher in this case. In both Tables 3 and 4, the numbers do not show clearly the zero and first orders of consistency, respectively, specially in the last columns;

Table 4 Local errors and values of $\tilde{p}$ for (31) with improved boundary conditions, $N=512$

\begin{tabular}{llllll}
\hline$\varepsilon$ & $\mathrm{M}=8$ & $\mathrm{M}=16$ & $\mathrm{M}=32$ & $\mathrm{M}=64$ & $\mathrm{M}=128$ \\
\hline $2^{-6}$ & $7.6949 \mathrm{E}-1$ & $4.5196 \mathrm{E}-1$ & $2.1054 \mathrm{E}-1$ & $8.0750 \mathrm{E}-2$ & $3.1093 \mathrm{E}-2$ \\
& 0.768 & 1.102 & 1.383 & 1.377 & \\
$2^{-8}$ & $8.0530 \mathrm{E}-1$ & $4.7209 \mathrm{E}-1$ & $2.1897 \mathrm{E}-1$ & $8.3539 \mathrm{E}-2$ & $3.4114 \mathrm{E}-2$ \\
& 0.770 & 1.108 & 1.390 & 1.292 & \\
$2^{-10}$ & $8.1665 \mathrm{E}-1$ & $4.7848 \mathrm{E}-1$ & $2.2157 \mathrm{E}-1$ & $8.4303 \mathrm{E}-2$ & $3.5206 \mathrm{E}-2$ \\
& 0.771 & 1.111 & 1.394 & 1.260 & \\
$2^{-12}$ & $8.2006 \mathrm{E}-1$ & $4.8039 \mathrm{E}-1$ & $2.2232 \mathrm{E}-1$ & $8.4499 \mathrm{E}-2$ & $3.5513 \mathrm{E}-2$ \\
& 0.772 & 1.112 & 1.396 & 1.251 & \\
$2^{-14}$ & $8.2105 \mathrm{E}-1$ & $4.8094 \mathrm{E}-1$ & $2.2253 \mathrm{E}-1$ & $8.4548 \mathrm{E}-2$ & $3.5592 \mathrm{E}-2$ \\
& 0.772 & 1.112 & 1.396 & 1.248 & \\
$2^{-16}$ & $8.2133 \mathrm{E}-1$ & $4.8110 \mathrm{E}-1$ & $2.2259 \mathrm{E}-1$ & $8.4561 \mathrm{E}-2$ & $3.5612 \mathrm{E}-2$ \\
& 0.772 & 1.112 & 1.396 & 1.248 & \\
- & - & - & - & - & - \\
& - & - & - & - & $3.5619 \mathrm{E}-2$ \\
$2^{-26}$ & $8.2144 \mathrm{E}-1$ & $4.8116 \mathrm{E}-1$ & $2.2261 \mathrm{E}-1$ & $8.4565 \mathrm{E}-2$ & \\
& 0.772 & 1.112 & 1.396 & 1.247 & \\
& & & & &
\end{tabular}


Table 5 Local errors and values of $\tilde{p}$ for (31) with natural boundary conditions, $\varepsilon=2^{-12}$

\begin{tabular}{llllll}
\hline $\mathrm{N}$ & $\mathrm{M}=8$ & $\mathrm{M}=16$ & $\mathrm{M}=32$ & $\mathrm{M}=64$ & $\mathrm{M}=128$ \\
\hline 1024 & $8.2025 \mathrm{E}-1$ & $5.7784 \mathrm{E}-1$ & $4.4430 \mathrm{E}-1$ & $3.0196 \mathrm{E}-1$ & $1.8190 \mathrm{E}-1$ \\
& 0.505 & 0.379 & 0.557 & 0.731 & \\
2048 & $8.2021 \mathrm{E}-1$ & $5.7923 \mathrm{E}-1$ & $4.4803 \mathrm{E}-1$ & $3.0573 \mathrm{E}-1$ & $1.8460 \mathrm{E}-1$ \\
& 0.502 & 0.371 & 0.551 & 0.728 & \\
\hline
\end{tabular}

these ones correspond to the largest values of $M$, where the fixed value of $N$ is not sufficiently large, and therefore, at these columns, in the estimated errors there is a substantial influence of the errors associated to the spatial discretization. To reduce this effect, in Tables 5 and 6, we show the numerical results obtained for a particular value of $\varepsilon$ when the discretization parameter $N$ is increased to $N=1024$ and $N=2048$; similar results are obtained for other values of $\varepsilon$. From them, we observe the corresponding numerical orders of consistency, closer to one in Table 6 as the theory predicts.

The second example that we consider is given by

$$
\begin{aligned}
& u_{t}-\varepsilon \Delta u+u_{x}+u_{y}+\left(5+2 t^{2} \exp \left(-1 /\left(\left(x-x^{2}\right)\left(y-y^{2}\right)\right)\right)\right) u \\
& =2 e^{-5 t}\left(\Psi(x) \Psi(y)-t^{2}\right),(x, y, t) \in \Omega \times[0,1], \\
& u(x, y, t)=e^{-5 t}(x+y-2 t), \text { in } \partial \times[0,1] \\
& u(x, y, 0)=x+y, \quad x, y \in[0,1] \text {, }
\end{aligned}
$$

where

$$
\Psi(z) \equiv z+\frac{e^{-1 / \varepsilon}\left(1-e^{z / \varepsilon}\right)}{1-e^{-1 / \varepsilon}}
$$

In this case, the exact solution is unknown.

We take again $m_{x}=m_{y}=1$ to define the piecewise uniform Shishkin mesh, and we decompose the source term by taking $f_{1}(x, y, t)=f_{2}(x, y, t)=f(x, y, t) / 2$.

Table 6 Local errors and values of $\tilde{p}$ for (31) with improved boundary conditions, $\varepsilon=2^{-12}$

\begin{tabular}{llllll}
\hline $\mathrm{N}$ & $\mathrm{M}=8$ & $\mathrm{M}=16$ & $\mathrm{M}=32$ & $\mathrm{M}=64$ & $\mathrm{M}=128$ \\
\hline 1024 & $8.2006 \mathrm{E}-1$ & $4.8037 \mathrm{E}-1$ & $2.2226 \mathrm{E}-1$ & $8.2802 \mathrm{E}-2$ & $2.9222 \mathrm{E}-2$ \\
& 0.772 & 1.112 & 1.424 & 1.503 & \\
2048 & $8.2006 \mathrm{E}-1$ & $4.8036 \mathrm{E}-1$ & $2.2224 \mathrm{E}-1$ & $8.2616 \mathrm{E}-2$ & $2.6699 \mathrm{E}-2$ \\
& 0.772 & 1.112 & 1.428 & 1.630 & \\
\hline
\end{tabular}


To approximate the maximum pointwise errors, we use a variant of the twomesh principle (see $[8,9]$ ). We calculate $\left\{\hat{u}^{N}\right\}$, the numerical solution on the mesh $\left\{\left(\hat{x}_{i}, \hat{y}_{j}, \hat{t}_{n}\right)\right\}$ containing the original mesh points and its midpoints, i.e.,

$$
\begin{aligned}
& \hat{x}_{2 i}=x_{i}, \quad i=0, \ldots, N, \quad \hat{x}_{2 i+1}=\left(x_{i}+x_{i+1}\right) / 2, \quad i=0, \ldots, N-1, \\
& \hat{y}_{2 j}=y_{j}, \quad j=0, \ldots, N, \quad \hat{y}_{2 j+1}=\left(y_{j}+y_{j+1}\right) / 2, \quad j=0, \ldots, N-1, \\
& \hat{t}_{2 m}=t_{m}, \quad m=0, \ldots, M, \quad \hat{t}_{2 m+1}=\left(t_{m}+t_{m+1}\right) / 2, \quad m=0, \ldots, M-1 .
\end{aligned}
$$

Then, the maximum errors at the mesh points of the coarse mesh are approximated by

$$
d_{i, j, N, M}=\max _{0 \leq m \leq M} \max _{0 \leq i, j \leq N}\left|u^{N}\left(x_{i}, y_{j}, t_{m}\right)-\hat{u}^{N}\left(x_{i}, y_{j}, t_{m}\right)\right|,
$$

and the orders of convergence are given by

$$
q=\log \left(d_{i, j, N, M} / d_{i, j, 2 N, 2 M}\right) / \log 2 .
$$

From the double-mesh differences in (33), we obtain the uniform maximum errors by

$$
d^{N, M}=\max _{\varepsilon} d_{i, j, N, M},
$$

and from them, in a usual way, the corresponding numerical uniform orders of convergence by

$$
q^{\text {uni }}=\log \left(d^{N, M} / d^{2 N, 2 M}\right) / \log 2 .
$$

Table 7 displays the results for problem (32) by using our method when classical boundary conditions are chosen. From them, we again observe a reduction in the order of uniform convergence.

Next, we evaluate the boundary conditions using (19) to solve the same problem. Table 8 displays the numerical results corresponding to this choice; from them, we observe smaller maximum errors, in comparison to Table 7 , and also we deduce a first order uniformly convergent behavior.

Table 7 Maximum errors and orders of convergence for (32) with natural boundary conditions

\begin{tabular}{llllll}
\hline$\varepsilon$ & $\mathrm{N}=16$ & $\mathrm{~N}=32$ & $\mathrm{~N}=64$ & $\mathrm{~N}=128$ & $\mathrm{~N}=256$ \\
$\mathrm{M}=8$ & $\mathrm{M}=16$ & $\mathrm{M}=32$ & $\mathrm{M}=64$ & $\mathrm{M}=128$ \\
\hline $2^{-6}$ & $5.9524 \mathrm{E}-2$ & $6.9820 \mathrm{E}-2$ & $5.3599 \mathrm{E}-2$ & $3.3555 \mathrm{E}-2$ & $1.9020 \mathrm{E}-2$ \\
& -.230 & 0.381 & 0.676 & 0.819 & \\
$2^{-8}$ & $6.5906 \mathrm{E}-2$ & $7.5892 \mathrm{E}-2$ & $5.7369 \mathrm{E}-2$ & $3.5686 \mathrm{E}-2$ & $2.0030 \mathrm{E}-2$ \\
& -.204 & 0.404 & 0.685 & 0.833 & \\
$2^{-10}$ & $6.7763 \mathrm{E}-2$ & $7.7925 \mathrm{E}-2$ & $5.8878 \mathrm{E}-2$ & $3.6511 \mathrm{E}-2$ & $2.0450 \mathrm{E}-2$ \\
& -.202 & 0.404 & 0.689 & 0.836 & \\
- & - & - & - & - & - \\
& - & - & - & - & $2.0677 \mathrm{E}-2$ \\
$2^{-26}$ & $6.8412 \mathrm{E}-2$ & $7.8680 \mathrm{E}-2$ & $5.9490 \mathrm{E}-2$ & $3.6912 \mathrm{E}-2$ & \\
& -.202 & 0.403 & 0.689 & 0.836 & \\
$d^{N, M}$ & $6.8412 \mathrm{E}-2$ & $7.8680 \mathrm{E}-2$ & $5.9490 \mathrm{E}-2$ & $3.6912 \mathrm{E}-2$ & $2.0677 \mathrm{E}-2$ \\
$q^{\text {uni }}$ & -.202 & 0.403 & 0.689 & 0.836 & \\
\hline
\end{tabular}


Table 8 Maximum errors and orders of convergence for (32) with improved boundary conditions

\begin{tabular}{|c|c|c|c|c|c|}
\hline$\varepsilon$ & $\mathrm{N}=16$ & $\mathrm{~N}=32$ & $\mathrm{~N}=64$ & $\mathrm{~N}=128$ & $N=256$ \\
\hline & $M=8$ & $M=16$ & $M=32$ & $M=64$ & $\mathrm{M}=128$ \\
\hline \multirow[t]{2}{*}{$2^{-6}$} & $3.4674 \mathrm{E}-2$ & $1.9782 \mathrm{E}-2$ & $1.0357 \mathrm{E}-2$ & $5.2911 \mathrm{E}-3$ & $2.6748 \mathrm{E}-3$ \\
\hline & 0.810 & 0.934 & 0.969 & 0.984 & \\
\hline \multirow[t]{2}{*}{$2^{-8}$} & $3.6338 \mathrm{E}-2$ & $2.0256 \mathrm{E}-2$ & $1.0613 \mathrm{E}-2$ & $5.4266 \mathrm{E}-3$ & $2.7390 \mathrm{E}-3$ \\
\hline & 0.843 & 0.932 & 0.968 & 0.986 & \\
\hline \multirow[t]{2}{*}{$2^{-10}$} & $3.6910 \mathrm{E}-2$ & $2.0575 \mathrm{E}-2$ & $1.0703 \mathrm{E}-2$ & $5.4676 \mathrm{E}-3$ & $2.7580 \mathrm{E}-3$ \\
\hline & 0.843 & 0.943 & 0.969 & 0.987 & \\
\hline \multirow[t]{2}{*}{-} & - & - & - & - & - \\
\hline & - & - & - & - & \\
\hline \multirow[t]{2}{*}{$2^{-26}$} & $3.7122 \mathrm{E}-2$ & $2.0764 \mathrm{E}-2$ & $1.0811 \mathrm{E}-2$ & $5.4988 \mathrm{E}-3$ & $2.7696 \mathrm{E}-3$ \\
\hline & 0.838 & 0.942 & 0.975 & 0.989 & \\
\hline$d^{N, M}$ & $3.7122 \mathrm{E}-2$ & $2.0764 \mathrm{E}-2$ & $1.0811 \mathrm{E}-2$ & $5.4988 \mathrm{E}-3$ & $2.7696 \mathrm{E}-3$ \\
\hline$q^{\text {uni }}$ & 0.838 & 0.942 & 0.975 & 0.989 & \\
\hline
\end{tabular}

Now, we estimate the local errors for this second example. As the exact solution is unknown, to approximate the errors, we proceed in a different way than before. To approximate $\tilde{U}_{N}^{n}$, we use one step of the fully discrete scheme given in (16), but replacing the numerical approximation at time $t_{n-1}, U_{N}^{n-1}$, by the numerical solution obtained on a very fine mesh of Shishkin type, with $N=1024, M=512$, which is denoted by $u_{1024,512}$. If $\bar{u}_{1024,512}$ is the piecewise bilinear interpolation of $u_{1024,512}$. We take again a large fixed value for spatial discretization parameter, concretely $N=$ 256 , in order to the errors in time dominate, then the local errors are approximated by

$$
\tilde{d}_{N, M}=\max _{0 \leq n \leq M} \max _{0 \leq i \leq N} \max _{0 \leq j \leq N}\left|\tilde{U}_{N}^{n}-\bar{u}_{1024,512}\left(x_{i}, y_{j}, t_{n}\right)\right|,
$$

and from them we calculate

$$
\tilde{q}=\log \left(\tilde{d}_{N, M} / \tilde{d}_{N, 2 M}\right) / \log 2,
$$

and the orders of consistency are given by $\tilde{q}-1$.

Table 9 Local errors and values of $\tilde{q}$ for (32) with natural boundary conditions, $N=256$

\begin{tabular}{llllll}
\hline$\varepsilon$ & $\mathrm{M}=8$ & $\mathrm{M}=16$ & $\mathrm{M}=32$ & $\mathrm{M}=64$ & $\mathrm{M}=128$ \\
\hline $2^{-6}$ & $2.3118 \mathrm{E}-1$ & $1.9211 \mathrm{E}-1$ & $1.2116 \mathrm{E}-1$ & $6.4267 \mathrm{E}-2$ & $2.9005 \mathrm{E}-2$ \\
& 0.267 & 0.665 & 0.915 & 1.148 & \\
$2^{-8}$ & $2.3903 \mathrm{E}-1$ & $1.9833 \mathrm{E}-1$ & $1.2524 \mathrm{E}-1$ & $6.6707 \mathrm{E}-2$ & $3.0369 \mathrm{E}-2$ \\
& 0.269 & 0.663 & 0.909 & 1.135 & \\
$2^{-10}$ & $2.4156 \mathrm{E}-1$ & $2.0036 \mathrm{E}-1$ & $1.2659 \mathrm{E}-1$ & $6.7566 \mathrm{E}-2$ & $3.0924 \mathrm{E}-2$ \\
& 0.270 & 0.662 & 0.906 & 1.128 & \\
& & & &
\end{tabular}


Table 10 Local errors and values of $\tilde{q}$ for (32) with improved boundary conditions, $N=256$

\begin{tabular}{llllll}
\hline$\varepsilon$ & $\mathrm{M}=8$ & $\mathrm{M}=16$ & $\mathrm{M}=32$ & $\mathrm{M}=64$ & $\mathrm{M}=128$ \\
\hline $2^{-6}$ & $7.0714 \mathrm{E}-2$ & $2.5038 \mathrm{E}-2$ & $7.5868 \mathrm{E}-3$ & $2.0084 \mathrm{E}-3$ & $4.6152 \mathrm{E}-4$ \\
& 1.498 & 1.723 & 1.917 & 2.122 & \\
$2^{-8}$ & $7.1447 \mathrm{E}-2$ & $2.5566 \mathrm{E}-2$ & $7.7610 \mathrm{E}-3$ & $2.0499 \mathrm{E}-3$ & $4.6930 \mathrm{E}-4$ \\
& 1.483 & 1.720 & 1.921 & 2.127 & \\
$2^{-10}$ & $7.1701 \mathrm{E}-2$ & $2.5793 \mathrm{E}-2$ & $7.8290 \mathrm{E}-3$ & $2.0662 \mathrm{E}-3$ & $7.2746 \mathrm{E}-4$ \\
& 1.475 & 1.720 & 1.922 & 1.506 & \\
\hline
\end{tabular}

Table 9 displays the local errors when boundary conditions (18) are taken, and Table 10 displays the local errors when (19) are considered. In order to not enlarge the paper in excess, we only show the results for three values of $\varepsilon$ which are sufficient to understand the differences between the two evaluations of the boundary conditions. In these tables, again the local errors for improved boundary conditions are smaller than for classical ones; also, the orders of consistency tend to zero for natural boundary conditions and to one for improved boundary conditions.

Acknowledgements The authors thank the referees their valuable suggestions which have helped to improve the presentation of this paper. This research was partially supported by the project MTM201452859 and the Diputación General de Aragón.

\section{References}

1. Alonso-Mallo, I., Cano, B., Jorge, J.C.: Spectral-fractional step Runge-Kutta discretizations for initial boundary value problems with time dependent boundary conditions. Math. Comp. 73, 1801-1825 (2004)

2. Bujanda, B., Clavero, C., Gracia, J.L., Jorge, J.C.: A high order uniformly convergent alternating direction scheme for time dependent reaction-diffusion singularly perturbed problems. Num. Math. 107, 1-25 (2007)

3. Clavero, C., Gracia, J.L., Jorge, J.C.: A uniformly convergent alternating direction HODIE finite difference scheme for 2D time dependent convection-diffusion problems. IMA J. Numer. Anal. 26, 155172 (2006)

4. Clavero, C., Gracia, J.L., O'Riordan, E.: A parameter robust numerical method for a two dimensional reaction-diffusion problem. Math. Comp. 74, 1743-1758 (2005)

5. Clavero, C., Jorge, J.C., Lisbona, F., Shishkin, G.I.: A fractional step method on a special mesh for the resolution of multidimensional evolutionary convection-diffusion problems. Appl. Num. Math. 27, 211-231 (1998)

6. Clavero, C., Jorge, J.C.: Another uniform convergence analysis technique of some numerical methods for parabolic singularly perturbed problem. Comp. Math. Appl. 70, 222-235 (2015)

7. Clavero, C., Jorge, J.C.: Uniform convergence and order reduction of the fractional implicit Euler method to solve singularly perturbed 2D reaction-diffusion problems. Appl. Math. Comp. 287-88, 12-27 (2016)

8. Farrell, P.A., Hegarty, A.: On the determination of the order of uniform convergence. In: Proceedings of IMACS'91 v. 2, pp. 501-502 (1991)

9. Farrell, P.A., Hegarty, A.F., Miller, J.J.H., O’ Riordan, E., Shishkin, G.I.: Robust computational techniques for boundary layers. Chapman and Hall (2000) 
10. Linss, T., Stynes, M.: Asymptotic analysis and Shishkin-type decomposition for an elliptic convectiondiffusion problem. J. Math. Anal. Appl. 261, 604-632 (2001)

11. Miller, J.J.H., O’Riordan, E., Shishkin, G.I.: Fitted numerical methods for singular perturbatiom problems, (revised edition). World Scientific (2012)

12. O'Riordan, E., Shishkin, G.I.: A technique to prove parameter-uniform convergence for a singularly perturbed convection-diffusion equation. J. Comput. Appl. Math. 206, 136-145 (2007)

13. Ostermann, A., Roche, M.: Runge-Kutta methods for partial differential equations and fractional orders of convergence. Math. Comp. 59, 403-420 (1992)

14. Roos, H.G., Stynes, M., Tobiska, L.: Robust numerical methods for singularly perturbed differential equations, (second edition). Springer-Verlag (2008)

15. Sanz-Serna, J.G., Verwer, J., Hundsdorfer, W.H.: Convergence and order reduction of Runge-Kutta schemes applied to evolutionary problems in partial differential equations. Numer. Math. 50, 405-418 (1986)

16. Shishkin, G.I.: Approximation of the solution to singularly perturbed boundary value problems with boundary layers. U.S.S.R. Comput. Maths. Math. Phys. 29, 1-10 (1989)

17. Shishkin, G.I., Shsihkina, L.P.: Difference methods for singular perturbation problems. Chapman \& Hall/CRC (2009)

18. Yanenko, N.N.: The method of fractional steps. Springer (1971) 